\title{
MAPEAMENTO E QUANTIFICAC̃̃̃O DE PARÂMETROS BIOFÍSICOS E RADIAÇÃO LÍQUIDA EM ÁREA DE ALGODOEIRO IRRIGADO
}

\author{
Mapping and quantification of biophysical parameters \\ and net radiation over irrigated cotton fields
}

\author{
Valéria Peixoto Borges ${ }^{1}$, Aureo Silva de Oliveira ${ }^{2}$, Bernardo Barbosa da Silva ${ }^{3}$
}

\begin{abstract}
RESUMO
O sensoriamento remoto tem se mostrado eficaz na avaliação de fluxos de energia e de propriedades biofísicas de superfícies vegetadas em escala regional. No presente trabalho, utilizou-se o algoritmo SEBAL - Surface Energy Balance Algorithm for Land e imagens TM - Landsat 5 para mapeamento e quantificação do albedo $(\alpha)$, NDVI, temperatura da superfície (T) e radiação líquida (R $)$ em área de algodão irrigado por pivô central, na Fazenda Busato $\left(13,25^{\circ} \mathrm{S} ; 43,42^{\circ} \mathrm{W} ; 436 \mathrm{~m}\right)$, município de Bom Jesus da Lapa, Bahia. Seis imagens de céu limpo ao longo do período da cultura (janeiro a agosto de 2007) e os respectivos dados meteorológicos foram utilizados para implementação do algoritmo. Após o processamento digital das imagens, verificou-se nítida relação dos parâmetros $\alpha, \mathrm{T}_{\mathrm{s}}$ e NDVI com o desenvolvimento da cultura. Os menores valores de $\alpha(10$ a $20 \%)$ e $\mathrm{T}\left(\leq 24^{\circ} \mathrm{C}\right)$ e os maiores de NDVI $(\geq 0,75)$ ocorreram na fase de máxima cobertura do solo. A radiação líquida $\left(\mathrm{R}_{\mathrm{n}}\right)$ diminuiu progressivamente com o tempo, influenciada, principalmente, pela diminuição da radiação solar incidente com o aumento do ângulo zenital. Os valores de $\mathrm{R}$ variaram de $430 \mathrm{~W} \mathrm{~m} \mathrm{a}^{-2} 700 \mathrm{~W} \mathrm{~m}^{-2}$ nos pivos cultivados. A técnica de sensoriamento empregada capturou de forma nítida a variabilidade temporal e espacial de $\mathrm{R}_{\mathrm{n}} \mathrm{e}$ dos parâmetros biofísicos, cujos valores encontrados são compatíveis com os reportados na literatura para a mesma cultura sob regime de irrigação.
\end{abstract}

Termos para indexação: Albedo, NDVI, balanço de radiação, SEBAL.

\section{ABSTRACT}

Remote sensing is currently an important tool for evaluation of net radiation and biophysical parameters over vegetated surfaces on a regional scale. In this research, the SEBAL - Surface Energy Balance Algorithm for Land and TM - Landsat 5 images were used to map and quantify the albedo $(\alpha)$, NDVI, surface temperature $\left(T_{s}\right)$ and net radiation $\left(R_{n}\right)$ of center-pivot irrigated cotton fields in the Busato Farm (13.25 $\mathrm{S} ; 43.42^{\circ} \mathrm{W} ; 436 \mathrm{~m}$ asl), western of State of Bahia, Brazil. Images from six clear-sky days during the cropping season (January to August 2007) and the corresponding meteorological data were used to run SEBAL. Results showed a clear relationship between $\alpha, \mathrm{NDVI}$, and $\mathrm{T}_{\mathrm{s}}$ and crop development. The lowest values of $\alpha(10$ to $20 \%)$ and $\mathrm{T}_{\mathrm{s}}\left(\leq 24^{\circ} \mathrm{C}\right)$, as well as the highest values of NDVI $(\geq 0.75)$, occurred at the time of maximum ground cover. Net radiation decreased progressively with time, following the decrease of the incident solar radiation with increasing solar zenith angle. In the cropped center pivots, $R_{n}$ ranged from $430 \mathrm{Wm}^{-2}$ to $700 \mathrm{Wm}^{-2}$. The remote sensing technique used in this study was consistent in capturing the temporal and spatial variability of the biophysical parameters and net radiation over the irrigated cotton, and their values are in agreement with those reported in the literature for the same crop in irrigated areas.

Index terms: Albedo, NDVI, radiation balance, SEBAL.

\section{(Recebido em 14 de janeiro de 2009 e aprovado em 16 de dezembro de 2009)}

\section{INTRODUÇÃO}

A energia disponível à superfície para os processos de aquecimento do ar e do solo, fotossíntese e evaporação da água advém do saldo das trocas radiativas de onda curta e de onda longa. A disponibilidade de radiação solar global, e, por conseguinte, do saldo de radiação, tem aplicações em vários campos de estudo, a exemplo nas atividades agropecuárias (Cargnelutti Filho et al., 2007), na hidrologia e na produtividade primária de comunidades vegetais. No manejo da água de irrigação, o saldo de radiação à superfície é um dos elementos mais relevantes no cálculo da demanda hídrica das culturas (Allen et al., 1998).

A determinação do saldo de radiação pode ser feita medindo-se ou estimando-se os componentes de onda curta e onda longa da radiação. As trocas radiativas à superfície e, por consequência, a magnitude do saldo de radiação, dependem da inclinação dos raios solares bem como das propriedades espectrais da superfície que, por sua vez, dependem do tipo de vegetação e do grau de cobertura e do tipo de solo (Campbell \& Diak, 2005).

$\mathrm{O}$ uso do sensoriamento remoto tem-se destacado como uma das alternativas para estimativa de fluxos radiativos e não-radiativos à superfície (Baastianssen, 2000; Bisht et al., 2005). Uma de suas maiores vantagens é permitir a determinação de forma direta, de parâmetros biofísicos e componentes dos balanços de radiação e de energia, sem a necessidade de conhecimento a priori do solo, cultura, e condições de manejo (Baastianssen et al., 2005). Ademais,

\footnotetext{
1Universidade Federal de Campina Grande/UFCG - Unidade Acadêmica de Ciências Atmosféricas - Av. Aprígio Veloso, 882 - Bodocongó - $58109-970$ Campina Grande, PB - valpborges@gmail.com

${ }^{2}$ Universidade Federal do Recôncavo da Bahia/UFRB - Núcleo de Engenharia de Água e Solo - Cruz das Almas, BA

3Universidade Federal de Campina Grande/UFCG - Unidade Acadêmica de Ciências Atmosférica - Campina Grande, PB
} 
técnicas de sensoriamento remoto e geoprocessamento propiciam maior freqüência de atualização dos dados, agilidade no processamento destes e são economicamente viáveis (Menezes et al., 2009).

Dados espectrais obtidos por sensores a bordo de satélites e armazenados em formato digital, das regiões do visível e do infravermelho (refletido e termal) do espectro eletromagnético, são utilizados para obtenção do saldo de radiação pixel-a-pixel (Allen et al., 2002). Adicionalmente, por serem métodos não-invasivos, técnicas de sensoriamento remoto com imagens de satélite, também possibilitam a avaliação de parâmetros biofísicos da vegetação, a exemplo do índice de área foliar (IAF), do índice de vegetação da diferença normalizada (NDVI) e do índice de vegetação ajustado para o solo (SAVI), frequentemente utilizados como indicadores de relativa abundância e atividade da vegetação (Jensen, 2000).

Técnicas de sensoriamento para avaliação do balanço de radiação e seus componentes em áreas agrícolas irrigadas do Pólo Juazeiro-Petrolina foram empregadas por Silva et al. (2005a,b). Os autores utilizaram o algoritmo SEBAL - Surface Energy Balance Algorithm for Land (Bastiaanssen et al., 1998) e imagens obtidas pelo sensor TM (Thematic Mapper) do satélite Landsat 5. O SEBAL é um algoritmo desenvolvido para estimar os balanços de radiação e de energia à superfície, por meio de imagens multiespectrais de satélites que contenham o canal termal e com demanda mínima de dados de superfície. Além do
Brasil, vários estudos em diferentes partes do mundo (Bastiaanssen, 2000; Bezerra, 2006; Bashir et al., 2008) vêm demonstrando a precisão e praticidade do SEBAL na avaliação dos fluxos de energia e massa no sistema soloplanta-atmosfera, com ênfase em áreas irrigadas.

Conduziu-se este trabalho, com o objetivo de mapear e quantificar parâmetros biofísicos e radiação líquida, em área de algodão irrigado por pivô central na sub-bacia do Rio Corrente (Bacia do São Francisco, Bahia), utilizando o algoritmo SEBAL e imagens de satélite.

\section{MATERIAL E MÉTODOS}

A área de estudo (Fazenda Busato) fica $35 \mathrm{~km}$ a oeste da cidade de Bom Jesus da Lapa (1315'18', S, 4325'05' W, $436 \mathrm{~m}$ ), Bahia. Detalhes da área são apresentados na Figura 1. O tipo climático, segundo Köppen é BSwh' (Superintendência de Estudos Econômicos e Sociaias da Bahia-SEI, 1998), caracterizado por quente de caatinga, com chuvas de verão e período seco bem definido no inverno, e ausência de excedente hídrico. As médias anuais de temperatura, precipitação e evapotranspiração potencial são, respectivamente, $25,3^{\circ} \mathrm{C}, 831 \mathrm{~mm}$ e $1418 \mathrm{~mm}$. Na região predominam Latossolos e Cambissolos, com vegetação de contato caatinga-floresta estacional.

Na época deste estudo (primeiro semestre de 2007), a propriedade possuía 2500 ha de algodão irrigado por pivô central, com as cultivares Delta Penta nos pivôs 20
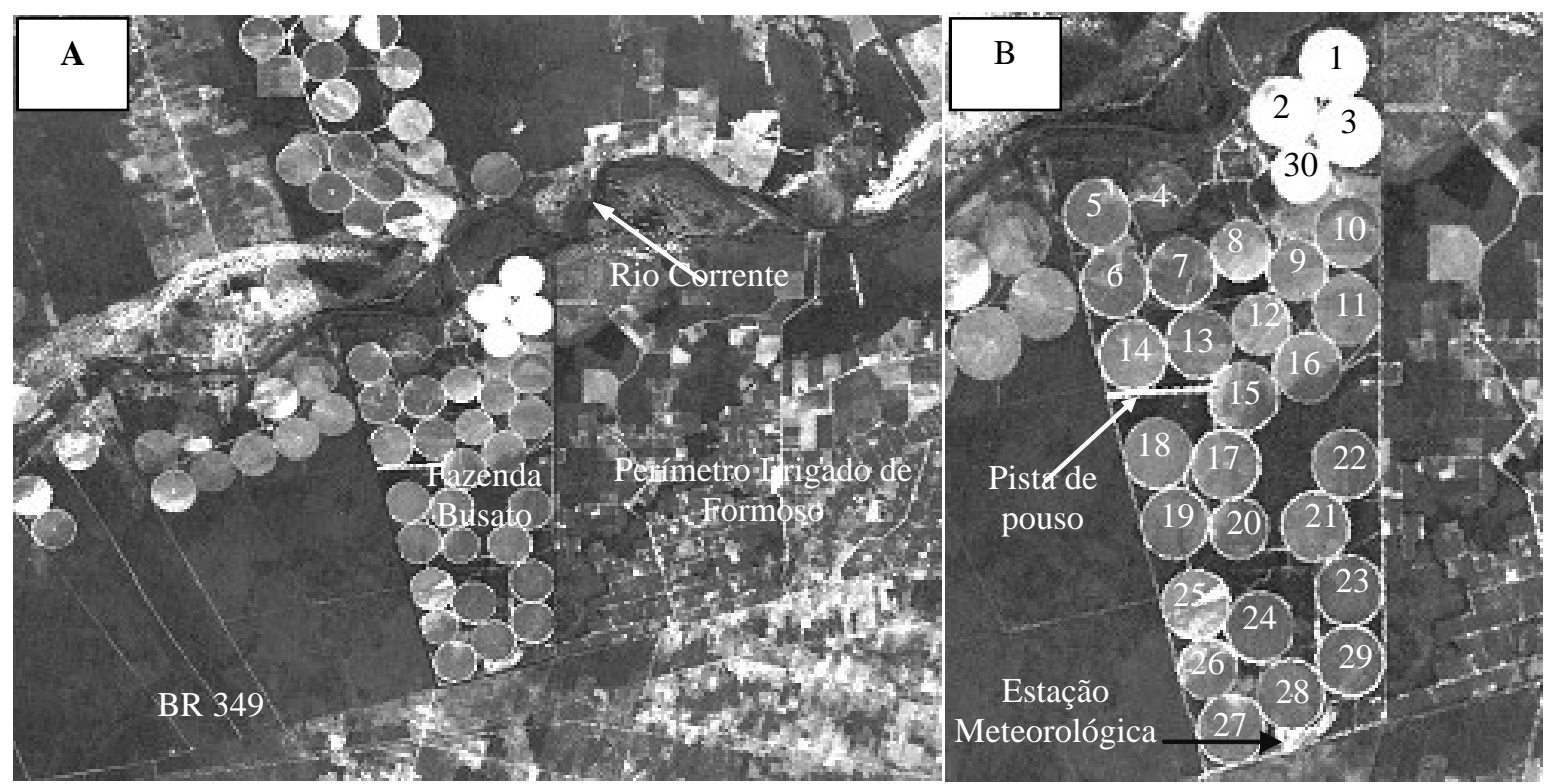

Figura 1 - Imagem da área de estudo em 26/04/07, obtida pelo sensor TM - Landsat 5. (A) Vista geral com localização da Fazenda Busato e (B) detalhe da fazenda com os pivôs de algodoeiro irrigado (5 a 29), pivôs em pousio (1, 2, 3 e 30) e pivô desativado (4). 
$\left(\mathrm{P}_{20}\right), 27,21$, e 5 e Delta Opal nos demais. Nos limites da fazenda, onde não havia algodão, predominava vegetação natural de baixo porte. $O$ período de semeadura do algodão variou de 08/01 no $\mathrm{P}_{23}$ a $06 / 03$ no $\mathrm{P}_{22}$, e a colheita, começando pelo $\mathrm{P}_{13}$ em 01/07, estendeu-se até 30/08 $\left(\mathrm{P}_{22}\right)$. $\mathrm{Na}$ rotina da propriedade, o turno de rega era diário. Dados oriundos de uma estação meteorológica automática (Davis Instruments Inc., modelo Vantage Pro $\left.{ }^{\circledast}\right)^{4}$, instalada na fazenda, eram utilizados para o cálculo computadorizado da ET de referência (ET) via FAO Penman-Monteith (Allen et al., 1998) e definição da lâmina líquida de irrigação $\left(\mathrm{ET}_{\mathrm{o}} \cdot \mathrm{K}_{\mathrm{c}}\right)$ em cada pivô central, sendo $\mathrm{K}_{\mathrm{c}}$ o coeficiente de cultivo do algodão. A mesma estação forneceu os dados de superfície para o processamento digital das imagens.

$\mathrm{O}$ saldo de radiação à superfície $\left(\mathrm{R}_{\mathrm{n}}\right)$, por unidade de área e de tempo, foi determinado pela Equação 1, após contabilização de todos os fluxos radiativos de onda curta e de onda longa:

$$
R_{n}=R_{S \downarrow}-\alpha R_{S \downarrow}+R_{L \downarrow}-R_{L \uparrow}-\left(1-\varepsilon_{o}\right) R_{L \downarrow}
$$

onde $\mathrm{R}_{\mathrm{n}}=$ saldo de radiação $\left(\mathrm{Wm}^{-2}\right), \alpha=$ albedo da superfície, $\mathrm{R}_{\mathrm{S} \downarrow}=$ radiação solar incidente $\left(\mathrm{Wm}^{-2}\right), \mathrm{R}_{\mathrm{L} \downarrow}=$ radiação de onda longa incidente $\left(\mathrm{Wm}^{-2}\right), \mathrm{R}_{\mathrm{L} \uparrow}=$ radiação de onda longa emitida pela superfície $\left(\mathrm{Wm}^{-2}\right)$ e $\varepsilon_{\mathrm{o}}=$ emissividade da superfície.

Para a solução da Equação 1, utilizou-se o algoritmo SEBAL, a partir de imagens obtidas pelo sensor TM do Landsat 5 (TM-L5). A determinação do saldo de radiação à superfície constitui-se numa das etapas do SEBAL, a partir da qual é estimada a partição dos fluxos não-radiativos (calor latente e calor sensível). Informações adicionais sobre o número de bandas, resolução e coeficientes de calibração do sensor TM-L5, podem ser encontradas em Jensen (2000) e Chander \& Markham (2003).

No Sistema de Referência Path and Row, da série de satélites Landsat (Jensen, 2000), a área da Fazenda Busato corresponde às coordenadas: órbita 219 e linha 69 , com passagem do satélite às 9:56 h. Na seleção das imagens, priorizou-se aquelas sem nuvens e regularmente distribuídas ao longo do período de estudo, de forma a permitir melhor avaliação das variáveis biofísicas e radiação líquida de acordo com as distintas fases fenológicas da cultura. Segundo esses critérios, selecionaram-se seis imagens do ano de 2007, obtidas nos dias 20 de janeiro (Dia do Ano - DDA 20), 25 de março (DDA 84), 26 de abril (DDA 116), 12 de maio (DDA 132), 28 de maio (DDA 148) e 15 de julho (DDA 196). O processamento

\footnotetext{
${ }^{4} \mathrm{~A}$ menção de marcas e produtos comerciais objetiva apenas informar o leitor e não significa, por parte dos autores, expressa recomendação para uso.
}

foi feito em recorte da imagem contendo toda a área da Fazenda Busato, semelhante à Figura 1B. O recorte foi mantido constante em todas as imagens do período. Todas as etapas de solução do algoritmo para determinação de $\mathrm{R}_{\mathrm{n}}$ basearamse em procedimentos apresentados por Allen et al. (2002).

A primeira etapa do processamento digital das imagens consistiu na geração de cartas das seguintes variáveis biofísicas: albedo $(\alpha)$, NDVI, IAF, SAVI e temperatura da superfície evaporante $\left(\mathrm{T}_{\mathrm{s}}\right)$. Na segunda etapa, procederam-se aos cálculos dos fluxos de radiação de onda curta e de onda longa incidentes, ambas consideradas constantes para toda a cena, e a geração da carta de onda longa emitida pela superfície. Por fim, o saldo de radiação à superfície foi determinado pixel a pixel, segundo a Equação 1.

\section{RESULTADOS E DISCUSSÃO}

De acordo com as datas de semeadura e colheita do algodão, o ciclo da cultura variou de $182\left(\mathrm{P}_{20} \mathrm{e} \mathrm{P}_{21}\right)$ a 211 dias $\left(\mathrm{P}_{5}\right)$ para cv. Delta Penta (média de 193 dias) e de 164 $\left(\mathrm{P}_{13}\right)$ a 219 dias $\left(\mathrm{P}_{28}\right.$ - setor leste) para cv. Delta Opal (média de 180 dias). Na Tabela 1, confronta-se no período de cultivo (janeiro a agosto de 2007), as datas das imagens com as datas de semeadura e de colheita em todos os pivôs cultivados. Verifica-se que foi possível obter da área de estudo somente três cenas consecultivas sem nuvens (26/04 e 12 e 28/05), ou seja, espaçadas de 16 dias (resolução temporal do satélite). Nenhuma imagem dos meses de fevereiro, junho e agosto puderam ser aproveitadas.

Da análise da Tabela 1, verifica-se que o plantio ocorreu predominantemente no mês de janeiro, dividindo-se as colheitas entre os meses de julho e agosto. Portanto, para a maioria dos pivôs, no intervalo entre março e junho, as plantas apresentavam avançado desenvolvimento vegetativo e reprodutivo. Segundo Rosolem (2007), é entre o surgimento da primeira flor (70 DAE - dias após a emergência) e o primeiro capulho (90 DAE), que as plantas atingem a altura máxima e ocorre o fechamento da copa (máxima interseção de luz). A fase final, da abertura do primeiro capulho à colheita, tem duração de 30 a 45 dias (Rosolem, 2007).

Apesar de ter predominado no mês de janeiro, o plantio distribuiu-se ao longo do mês, com intervalo de até 20 dias. Esse fato, associado a outras fontes de variabilidade, como implantação de dois cultivares de algodão, diferenças nas lâminas de irrigação aplicadas, ocorrência de nematóides, entre outras, podem ter afetado a resposta espectral da cobertura vegetal. Cartas em escala de cinza, seguindo ordem crescente de valores, dos tons mais escuros aos mais claros, são apresentadas evidenciando a variação espacial e temporal da radiação líquida $\left(\mathrm{R}_{\mathrm{n}}\right)$ e alguns parâmetros biofísicos (albedo, NDVI e temperatura da superfície). 
Tabela 1 - Datas de obtenção das imagens TM - Landsat 5 (órbita/linha: 219/69), de semeadura e de colheita (entre parênteses) do algodão no primeiro semestre de 2007, na Fazenda Busato, oeste da Bahia.

\begin{tabular}{|c|c|c|c|}
\hline Mês & $\begin{array}{l}\text { Data da } \\
\text { imagem }\end{array}$ & Pivô e data de semeadura & Pivô e data de colheita \\
\hline Jan & 20 & $\begin{array}{c}23(8)^{*} ; 28(9) ; 15 \text { e } 17(11) ; 12(12) ; 10-\mathrm{N}^{* *} \\
(15) ; 24-\mathrm{O}, 26 \text { e } 29(16) ; 14(17) ; 6 \text { e } 13(18) ; \\
7,8-S \text { e } 24-\mathrm{L}(19) ; 5(20) ; 20 \text { e } 21(21) ; 19 \\
(22) ; 18(23) ; 27(24) ; 16(26) ; 9 \text { e } 10-\mathrm{S}(27) ; \\
11(28)\end{array}$ & - \\
\hline Fev & - & $25(27)$ & - \\
\hline Mar & 25 & $8-\mathrm{N}(1) ; 22(6)$ & - \\
\hline Abr & 26 & - & - \\
\hline Mai & 12 e 28 & - & - \\
\hline Jun & - & - & - \\
\hline Jul & 15 & - & $\begin{array}{l}13(1) ; 7(4) ; 12(6) ; 14(8) ; 15(10) ; 16(11) ; \\
10-\mathrm{S}(13) ; 10-\mathrm{N}(14) ; 17 \text { e } 18(19) ; 19(21) ; 20 \\
\quad \text { e } 21(22) ; 6(23) ; 11(27) ; 23(28)\end{array}$ \\
\hline Ago & - & - & $\begin{array}{c}\text { 8-S (4); } 5 \text { e } 28-\mathrm{O}(7) ; 9(9) ; 24-\mathrm{O}(11) ; 24-\mathrm{L} \\
(13) ; 29(14) ; 28-\mathrm{L}(16) ; 26(18) ; 27(23) ; 25 \\
(26) ; 8-\mathrm{N}(27) ; 22(30)\end{array}$ \\
\hline
\end{tabular}

* dia do plantio ou colheita entre parênteses; ${ }^{* *}$ setor do pivô $(\mathrm{N}=$ norte, $\mathrm{S}=$ sul, $\mathrm{L}=$ Leste, e $\mathrm{O}=$ oeste $)$

Na Figura 2, mostra-se tendência de decréscimo do albedo $(\alpha)$ com o desenvolvimento da cultura, em virtude de maior interceptação e absorção da energia incidente, em razão do aumento da densidade foliar, o que pode ser corroborado pelo índice de vegetação da diferença normalizada (NDVI) determinado no mesmo período, apresentado mais à frente na Figura 3. Na data da primeira imagem (20/01), quando havia menor cobertura vegetal, o albedo nas áreas dos pivôs esteve entre 20 e 30\% (Figura 2A). Em alguns pivôs com $\alpha$ inferior a $15 \%$ nesta data, verificouse a existência de restos de cultura de safra anterior cobrindo o solo.

Nos meses de abril a maio (maior cobertura do solo), o albedo, em praticamente todos os pivôs, variou de 10 a $20 \%$, sendo que alguns casos, principalmente por ocasião da primeira passagem do satélite no mês de maio, o albedo variou de 10 a $15 \%$. Observa-se em todas as cenas que o albedo variou de pivô para pivô e também num mesmo pivô. Diferenças nas datas de plantio e manejo da cultura (fertilidade do solo, irrigação, pragas e doenças, etc) respondem pelas diferenças nas respostas espectrais. Valores semelhantes de albedo para outras culturas agrícolas irrigadas, incluindo o algodão, foram relatados por Costa Filho (2005) e Silva et al. (2005a,b) em estudos também com sensoriamento remoto. Kustas et al. (1990) obtiveram albedo de $22 \%$ em cultivo de algodão irrigado.
Na Figura 3, mostra-se a evolução do índice de vegetação da diferença normalizada (NDVI) em toda a área cultivada e a variação espacial do NDVI num mesmo pivô central bem como entre pivôs em distintas fases do ciclo.

Na cena de 20/01 (Figura 3A), a maioria dos pivôs apresentou NDVI inferior a 0,2, valor compatível com áreas de vegetação rala ou solo exposto. O NDVI é um indicador de relativa abundância e atividade da vegetação verde (Jensen, 2000) e diferentemente do albedo, apresentou tendência de crescimento com o desenvolvimento da cultura. Os maiores valores de NDVI (superiores a 0,75 ) ocorreram no intervalo de 26/04 a 28/05, no qual, em muitos pivôs, a cultura já se encontrava em fases fenológicas indicativas de máxima cobertura do solo (emissão de flores, formação de maçãs e capulhos). Na cena de 28/05, o NDVI ficou acima de 0,50 em todos pivôs cultivados.

Os picos de NDVI coincidiram com os do índice de área foliar (IAF), parâmetro biofísico estimado via SEBAL, por meio do índice SAVI. O valor máximo do IAF ficou em torno de 6,0, e segundo Rosolem (2007), os maiores valores de IAF para o algodoeiro ocorrem na fase de floração, coerente com Doorenbos \& Kassam (1979). Bezerra (2006) registrou NDVI igual a 0,70 para algodão irrigado nas imediações da Chapada do Araripe, Ceará. Com base numa série temporal de imagens do sensor MODIS de área irrigada no Uzbequistão, Conrad et al. (2007) determinaram a curva 
de NDVI para a cultura do algodão, evidenciando um mínimo em torno de 0,12 e um máximo de 0,60.

Com imagens do sensor TM-L5, a temperatura da superfície $\left(\mathrm{T}_{\mathrm{s}}\right)$ é estimada a partir da banda termal (canal 6). A cobertura vegetal foi o principal fator de variação de $\mathrm{T}$ (Figura 4). Em janeiro, as temperaturas registradas no momento da passagem do satélite foram maiores, inclusive nas áreas dos pivôs, onde $\mathrm{T}_{\mathrm{s}}$ variou de $30 \mathrm{a} 40^{\circ} \mathrm{C}$. Com o desenvolvimento da cultura, foi possível identificar áreas circulares em tons mais escuros (menores temperaturas), delimitando claramente os pivôs. Nos meses em que o algodoeiro apresentou máximo NDVI (abril e maio), as temperaturas nos pivôs não ultrapassaram $24^{\circ} \mathrm{C}$, resultado do acúmulo de massa verde e frequente suprimento de água (irrigações diárias), potencializando a transpiração das plantas.

Quando a cobertura do solo é máxima e não há restrição hídrica, $\lambda E T$ (fluxo de calor latente) pode representar até $70 \%$ do saldo de radiação, refrigerando o dossel vegetativo (Allen et al., 1998). Com a aproximação da época de colheita e senescência das plantas (cena de 15/07) as temperaturas da superfície já se apresentavam maiores.

A radiação de onda longa emitida por uma superfície $\left(\mathrm{R}_{\mathrm{L} \uparrow}\right)$ é função da sua temperatura absoluta e emissividade, de acordo com a equação de Stefan-Boltzman (Jensen, 2000). Portanto, variações de $\mathrm{R}_{\mathrm{L} \uparrow}$ na área de estudo estiveram condicionadas a mudanças na cobertura vegetal pela cultura do algodão nos pivôs e adjacências abrangidas pelo recorte da cena (Figura 1). A emissão de onda longa é um componente subtrativo do saldo de radiação, portanto, quanto menor $\mathrm{R}_{\mathrm{L}}$. maior a energia disponível para os fluxos não-radiativos, como a evapotranspiração. Na cena de $20 / 01$, a $\mathrm{R}_{\mathrm{L} \uparrow}$ variou de 420 a $430 \mathrm{Wm}^{-2}$ na água e acima de $470 \mathrm{Wm}^{-2} \mathrm{em}$ pivôs ainda não semeados. Os menores valores de $\mathrm{R}_{\mathrm{L} \uparrow}$ tanto na água (401 a $410 \mathrm{Wm}^{-2}$ ), quanto no algodão irrigado (407 a $417 \mathrm{Wm}^{-2}$ ) ocorreram em 12/05, período de alto NDVI (Figura 3). Esses valores estão de acordo com os obtidos por Silva et al. (2005b), que encontraram $\mathrm{R}_{\mathrm{L} \uparrow}$ em torno de $495 \mathrm{Wm}^{-2}$ em solo exposto e até $458 \mathrm{Wm}^{-2}$ em área de fruteiras irrigadas.

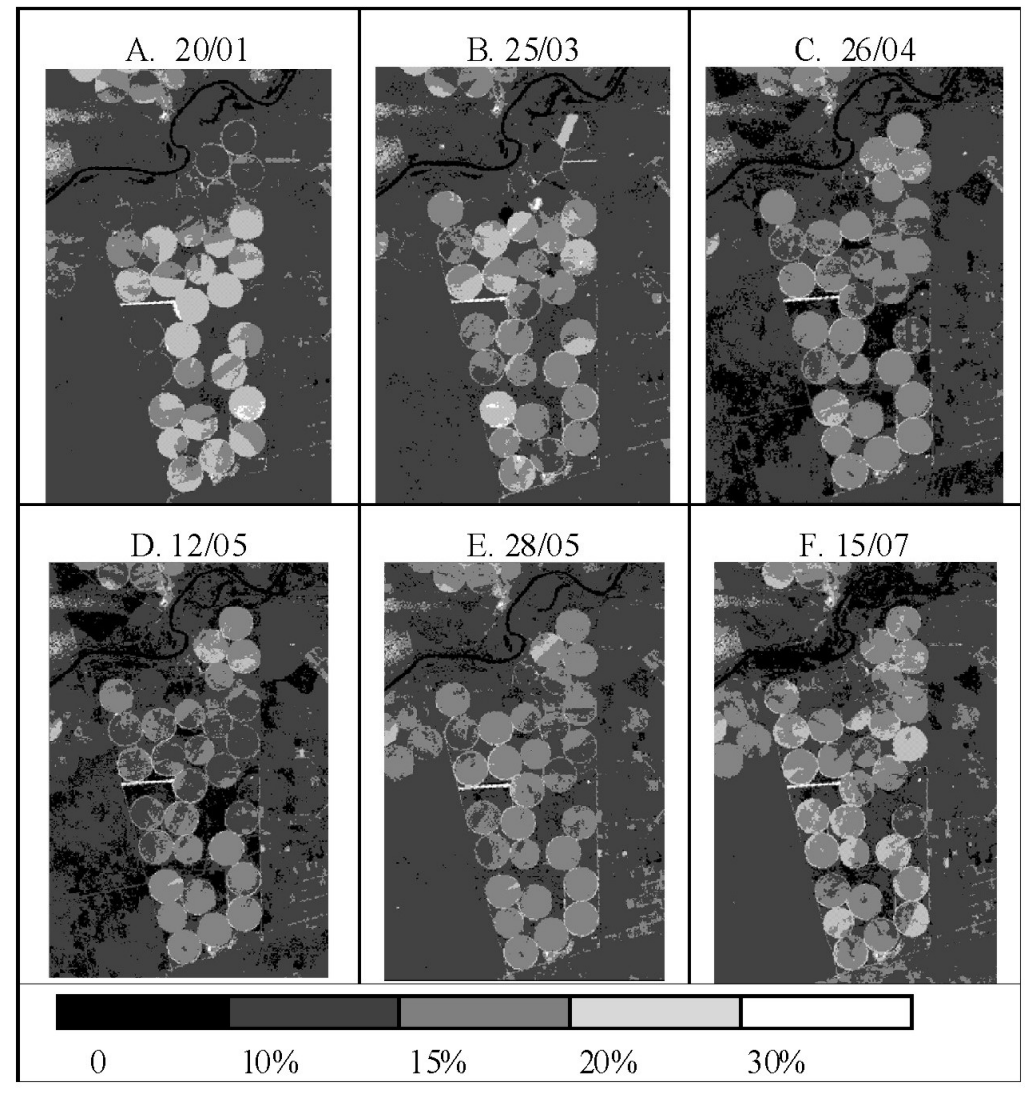

Figura 2 - Variação espacial e temporal do albedo, $\alpha$, para os dias 20/01, 25/03, 26/04, 12/05, 28/05 e 15/07 de 2007, em área de algodoeiro irrigado, Fazenda Busato, oeste da Bahia. 


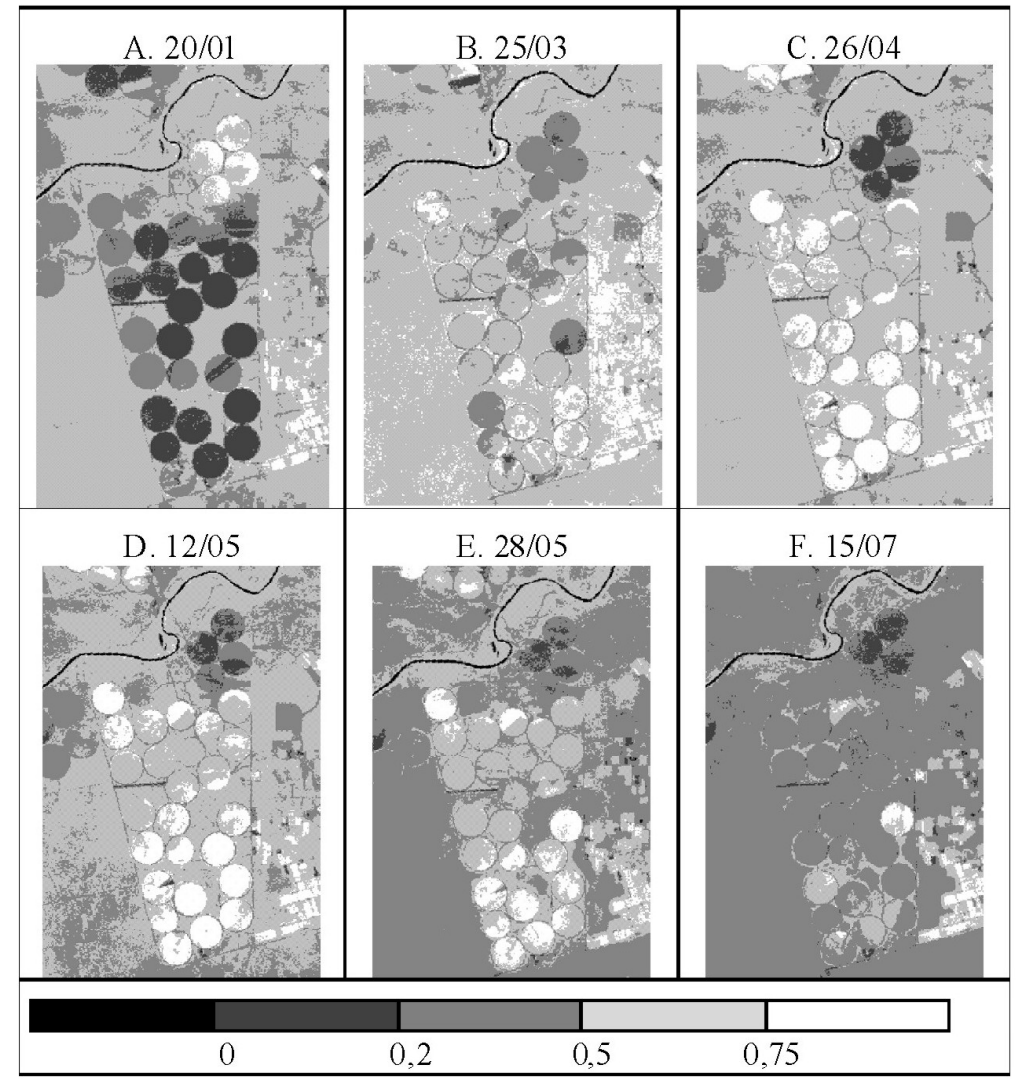

Figura 3 - Variação temporal e espacial do NDVI para os dias 20/01, 25/03, 26/04, 12/05, 28/05 e 15/07 de 2007, em área de algodoeiro irrigado, Fazenda Busato, oeste da Bahia.

As cartas de radiação líquida $\left(\mathrm{R}_{\mathrm{n}}\right)$ são apresentadas na Figura 5. Em razão do reduzido albedo da água, a máxima radiação líquida à superfície ocorreu, em todas as datas, no leito do rio Corrente, com valores entre $550 \mathrm{Wm}^{-2} \mathrm{em}$ julho e $809 \mathrm{Wm}^{-2}$ em janeiro. Os menores registros de $\mathrm{R}_{\mathrm{n}}$ foram detectados em solo totalmente exposto (pista de pouso, por exemplo) ou com vegetação rala (pivôs em pousio, antes do plantio e durante a colheita). Nesses pixels, registrou-se em julho (Figura 5F), os menores valores de $\mathrm{R}_{\mathrm{n}}$, da ordem de $305 \mathrm{Wm}^{-2}$. Conforme a Equação 1, albedo e $\mathrm{R}_{\mathrm{L} \uparrow}$ são componentes subtrativos do saldo de radiação. Os pixels acima mencionados, em decorrência de pouca ou ausente cobertura vegetal, apresentaram alto albedo e emitiram mais radiação terrestre que os demais, o que resultou em saldo de radiação menor em comparação às outras superfícies imageadas nas mesmas datas.

A radiação líquida instantânea nos pivôs cultivados variou de $430 \mathrm{Wm}^{-2}$ na cena de 28/05 (Figura 5E) a $700 \mathrm{Wm}^{-2}$ na cena de 25/03 (Figura 5B), apresentando tendência de decréscimo durante o período de estudo. A tendência de diminuição de $\mathrm{R}_{\mathrm{n}}$ com o tempo acompanhou o decréscimo observado na radiação solar incidente $\left(R_{S \downarrow}\right)$, estimada em função do cosseno do ângulo zenital, dia do ano, transmissividade atmosférica e constante solar $\left(1367 \mathrm{Wm}^{-2}\right.$ ) (Allen et al., 2002). O ângulo zenital aumentou de 20/01 a 15/07, provocando redução em $R_{S \downarrow}$ para dia de céu claro, de $913 \mathrm{Wm}^{-2}$ para $698 \mathrm{Wm}^{-2}$, respectivamente, no instante de passagem do satélite. Essa redução, por conseguinte, teve efeito significativo sobre o decréscimo observado nos valores de $R_{n}$, mesmo nos períodos de menor albedo (Figura 5C e Figura 5D) quando, de acordo com a fenologia da cultura (Rosolem, 2001), as plantas estavam na fase de floração/emissão de maçãs na maior parte dos pivos, com exceção dos pivôs 25,22 e 8 -N que tiveram plantios em fevereiro e março. No mês de julho, a redução em $R_{n}$ foi ainda potencializada pelo aumento do albedo (Figura 2) e aumento da temperatura da superfície (Figura 4), em virtude das primeiras colheitas. 


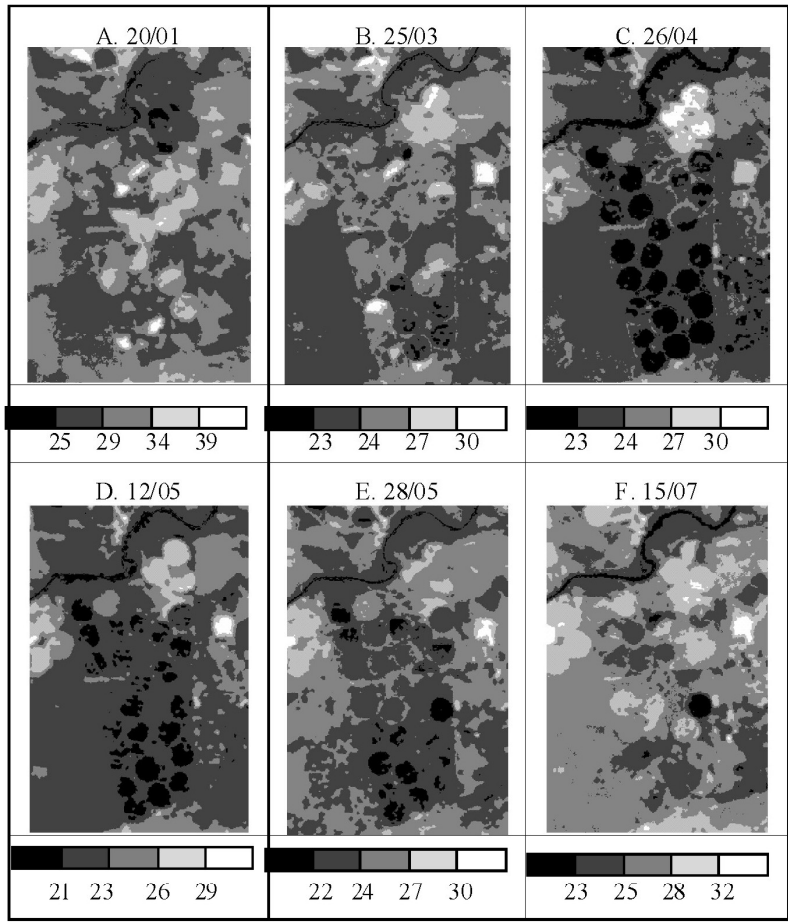

Figura 4 - Variação temporal e espacial da temperatura da superfície, $\mathrm{T}_{\mathrm{s}}\left({ }^{\circ} \mathrm{C}\right)$ para os dias 20/01, 25/03, 26/04, 12/05, 28/ 05 e 15/07 de 2007, em área de algodoeiro irrigado, Fazenda Busato, oeste da Bahia.

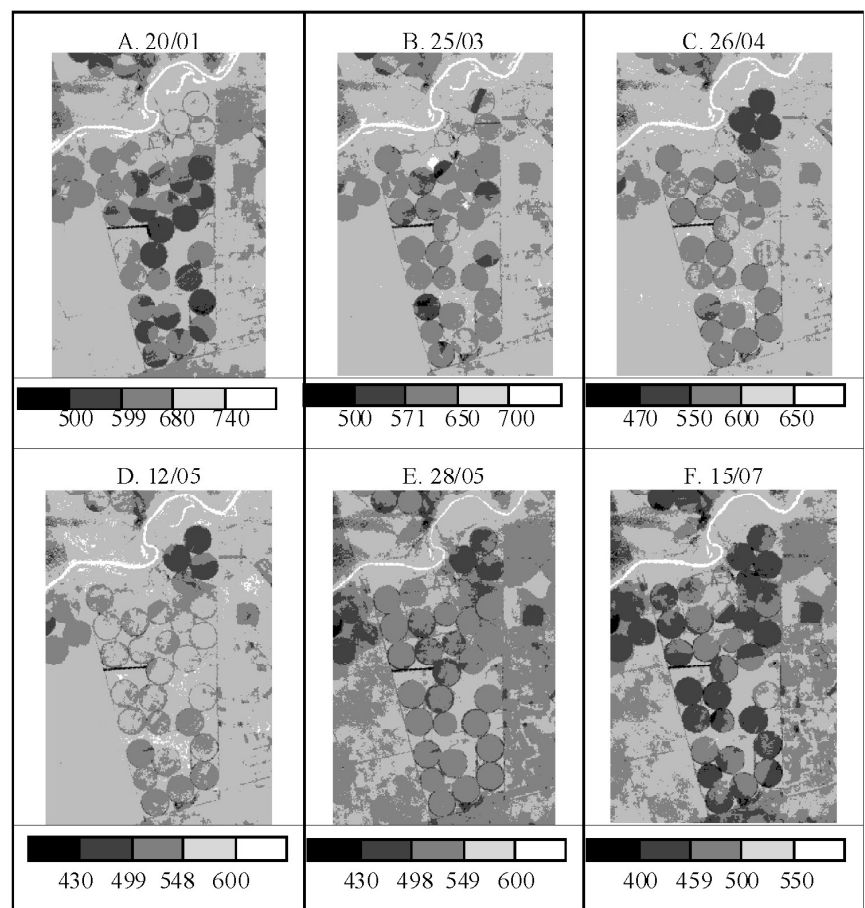

Figura 5 - Variação espacial e temporal da radiação líquida, $\mathrm{R}_{\mathrm{n}}\left(\mathrm{W} \mathrm{m}^{-2}\right)$ para os dias 20/01, 25/03, 26/04, 12/05, 28/05 e 15/07 de 2007, em área de algodoeiro irrigado, Fazenda Busato, oeste da Bahia. 
Os resultados encontrados para $\mathrm{R}_{\mathrm{n}}$ são compatíveis com os apresentados por outros autores. Chavez et al. (2007) obtiveram via SEBAL, $\mathrm{R}_{\mathrm{n}}$ entre 690 e $750 \mathrm{Wm}^{-2}$ em corpos d'água e áreas recentemente irrigadas; em pixels com alta biomassa sem déficit hídrico, $\mathrm{R}_{\mathrm{n}}$ variou de 600 a $650 \mathrm{Wm}^{-2} \mathrm{e}$ para solos descobertos, de 500 a $550 \mathrm{Wm}^{-2}$. Em região do semi-árido brasileiro, Bezerra (2006) encontrou $\mathrm{R}_{\mathrm{n}}$ entre 650 e $700 \mathrm{Wm}^{-2}$ em áreas com boa cobertura vegetal, incluindo áreas irrigadas. Em área de algodão BRS-201 irrigado, Borges (2002) obteve por meio de um saldo-radiômetro, $R_{n}$ da ordem de $600 \mathrm{Wm}^{-2}$ na fase de crescimento vegetativo da cultura e em torno de $700 \mathrm{Wm}^{-2}$, na fase reprodutiva. Bastiaanssen (2000), por outro lado, usando o SEBAL e imagens TM- L5, obteve para a cultura do algodão na Turquia e em duas datas distintas (26/06 e 29/08/1998), valores instantâneos médios de $\mathrm{R}_{\mathrm{n}}$ inferiores aos reportados neste estudo. Em 26/ 06, antes do início da temporada de irrigação e com o solo parcialmente coberto, $R_{n}$ foi de $374 \mathrm{Wm}^{-2}$ enquanto $R_{n}$ foi de $272 \mathrm{Wm}^{-2}$, após o término da estação de irrigação.

\section{CONCLUSÕES}

De maneira relevante, conclui-se que:

- a técnica de sensoriamento empregada capturou de maneira evidente, a variabilidade temporal e espacial dos parâmetros biofísicos albedo $(\alpha)$, NDVI e temperatura da superfície $\left(\mathrm{T}_{\mathrm{s}}\right.$ ) bem como a variabilidade da radiação líquida $\left(\mathrm{R}_{\mathrm{n}}\right)$ nos pivôs cultivados e áreas adjacentes;

- verificou-se, a partir do mapeamento, nítida relação dos parâmetros $\alpha$, NDVI e T com o grau de cobertura do solo pela cultura do algodão. Tanto $\alpha$ quanto $\mathrm{T}_{\mathrm{s}}$ diminuíram com o desenvolvimento da cultura, enquanto o NDVI aumentou;

- a radiação líquida diminuiu progressivamente com o ciclo da cultura, revelando-se mais sensível à redução na radiação solar incidente, do que às características da superfície;

- os valores de $\alpha$, NDVI, $\mathrm{T}_{\mathrm{s}}$ e $\mathrm{R}_{\mathrm{n}}$ encontrados por meio do SEBAL e imagens TM - Landsat 5, no oeste da Bahia para o algodão irrigado, são compatíveis com os de outros estudos com a mesma cultura e determinados pela mesma técnica de sensoriamento remoto.

\section{AGRADECIMENTOS}

Ao Sr. Hélio Busato, proprietário da Fazenda Busato e demais funcionários, pela permissão e apoio para realização desta pesquisa; ao CNPq pelo apoio financeiro por meio do Edital Universal 2006 (Proc. 475198/06-9); à FAPESB pelo Auxílio à Dissertação de Mestrado (Proc. APR 100/2007) e à CAPES pela concessão da bolsa de mestrado, nossos agradecimentos.

\section{REFERÊNCIAS BIBLIOGRÁFICAS}

ALLEN, R.G.; PEREIRA, L.S.; RAES, D.; SMITH, M. Crop evapotranspiration: guidelines for computing crop water requirements. Rome: UN-FAO, 1998. 300p.

(Irrigation and Drainage Paper, 56).

ALLEN, R.G.; WATERS, R.; TREZZA, R.; TASUMI, M.; BASTIAANSSEN, W. Sebal -surface energy balance algorithms for land: idaho implementation: advance training and users manual. Moscow: Idaho Department of Water Resources, 2002. 98p.

BASHIR, M.A.; HATA, T.; TANAKAMARU, H.; ABDELHADI, A.W.; TADA, A. Satellite-based energy balance model to estimate seasonal evapotranspiration for irrigated sorghum: a case study from the Gezira scheme, Sudan. Hydrology and Earth System Sciences, Katlenburg-Lindau, v.12, n.4, p.1129-1139, 2008.

BASTIAANSSEN, W.G.M. SEBAL-Based sensible and latent heat fluxes in the irrigated Gediz Basin, Turkey. Journal of Hydrology, Amsterdam, v.229, n.1/2, p.87-100, 2000 .

BASTIAANSSEN, W.G.M.; MENENTI, M.; FEDDES, R.A.; HOLTSLAG, A.A.M. Remote Sensing Surface Energy Balance Algorithm for Land (SEBAL): 1., formulation. Journal of Hydrology, Amsterdam, v.212/ 213, n.1/4, p.198-212, 1998.

BASTIAANSSEN, W.G.M.; NOORDMAN, E.J.M.; PELGRUM, H.; DAVIDS, G.; THORESON, B.P.; ALLEN, R.G. SEBAL model with remotely sensed data to improve water-resources management under actual field conditions. Journal of Irrigation and Drainage Engineering, New York, v.131, n.1, p.85-93, 2005.

BEZERRA, B.G. Balanço de energia e evapotranspiração em áreas com diferentes tipos de cobertura de solo no Cariri Cearense através do algoritmo SEBAL. 2006. 127f. Dissertação (Mestrado em Meteorologia)Universidade Federal de Campina Grande, Campina Grande, 2006.

BISHT, G.; VENTURINI, V.; ISLAM, S.; JIANG, L. Estimation of net radiation using MODIS (Moderate Resolution Imaging Spectroradiometer) data for clear sky days. Remote Sensing of Environment, Amsterdam, v.97, n.1, p.52-67, 2005. 
BORGES, P.F. Crescimento, desenvolvimento e balanço de radiação do algodoeiro herbáceo BRS - $201 \mathrm{em}$ condições irrigadas. 2002. 87f. Dissertação (Mestrado em Meteorologia)-Universidade Federal de Campina Grande, Campina Grande, 2002.

CAMPBELL, G.S.; DIAK, G.R. Net and thermal radiation estimation and measurement. In: VINEY, M.K.

Micrometeorology in agricultural systems. Madson: American Society of Agronomy/Crop Science Society of America/Soil Science of America, 2005. 584p. (Agronomy monograph, 47).

CARGNELUTTI FILHO, A.; MATZENAUER, R.; MALUF, J. R. T.; SILVA, B. P. da. Tamanho de amostra para a estimativa das médias decendiais de radiação solar global no Estado do Rio Grande do Sul. Ciência e Agrotecnologia, Lavras, v.31, n.5, p.1402-1410, set./out., 2007.

CHANDER, G.; MARKHAM, B. Revised Landsat-5 TM radiometric calibration procedures and postcalibration dynamic ranges. IEEE Transactions on Geoscience and Remote Sensing, New York, v.41, n.11, part 2, p.26742677, 2003.

CHAVEZ, J.L.; GOWDA, P.H.; MAREK, T.H.; NEW, L.L. Evapotranspiration mapping using METRICTM for region with highly advective conditions. In: ASABE ANNUAL INTERNATIONAL MEETING, 2007, Minneapolis. Anais... Minneapolis: ASABE, 2007.

CONRAD, C. et al. Mapping and assessing water use in a Central Asian irrigation system by utilizing MODIS remote sensing products. Irrigation and Drainage Systems, Dordrecht, v.21, n.3/4 , p.197-218, 2007.

COSTA FILHO, J.F. Avaliação dos componentes do balanço de energia e evapotranspiração em uma região semi-árida usando imagens orbitais Landsat-5 TM e Terra-Modis. 2005. 141f. Tese (Doutorado em Recursos Naturais)-Universidade Federal de Campina Grande, Campina Grande, 2005.
DOORENBOS, J.; KASSAM, A.H. Yield response to water. Roma: UN-FAO, 1979. 193p. (Irrigation and Drainage Paper, 33).

JENSEN, J.R. Remote sensing of the environment: an earth resource perspective. New Jersey: Prentice Hall, 2000. 544p.

KUSTAS, W.P.; MORAN, S.M.; JACKSON, R.D.; GAY, L.W.; DUELL, L.F.W.; KUNKEL, K.E.; MATTHIAS, A.D. Instantaneous and daily values of the surface energy balance over agricultural fields using remote sensing and a reference field in an arid environment. Remote Sensing of Environment, Amsterdam, v.32, n.2/3, p.125141, 1990.

MENEZES, M. D. de; CURI, N.; MARQUES, J. J.; MELLO, C. R. de; ARAÚJO, A. R. de. Levantamento pedológico e sistema de informações geográficas na avaliação do uso das terras em sub-bacia hidrográfica de Minas Gerais. Ciência e Agrotecnologia, Lavras, v.33, n.6, p.1544-1553, nov./dez., 2009.

ROSOLEM, C.A. Fenologia e ecofisiologia no manejo do algodoeiro. In: FREIRE, E.C. (Ed.). Algodão no Cerrado do Brasil. Brasília: Associação Brasileira dos Produtores de Algodão, 2007. 918p.

SILVA, B.B.; LOPES, G.M.; AZEVEDO, P.V. Balanço de radiação em áreas irrigadas utilizando imagens Landsat 5-TM. Revista Brasileira de Meteorologia, Rio de Janeiro, v.20, n.2, p.243-252, 2005a.

SILVA, B.B.; LOPES, G.M.; AZEVEDO, P.V. Determinação do albedo de áreas irrigadas com base em imagens Landsat 5-TM. Revista Brasileira de Agrometeorologia, Pelotas, v.13, n.2, p.201-211, 2005 b.

\section{SUPERINTENDÊNCIA DE ESTUDOS ECONÔMICOS E SOCIAS DA BAHIA. Atributos climáticos do Estado da Bahia. Salvador, 1998. 85p. (Série Estudos e Pesquisas, 38).}

